\title{
Endoscopic management of duodenal perforations caused by migrated biliary plastic stents $\square$
}

\section{다)(1) $\odot$}

Authors

Shin Hee Kim¹, Jong Ho Moon ${ }^{1}$, Yun Nah Lee ${ }^{1}$, Tae Hoon Lee ${ }^{2}$, Sang Myung Woo ${ }^{3}$, Woo Jin Lee ${ }^{3}$, Sung II Jang ${ }^{4}$, Dong Ki Lee $^{4}$

Institutions

1 Digestive Disease Center and Research Institutes, Department of Internal Medicine, SoonChunHyang University School of Medicine, Bucheon, Korea

2 Digestive Disease Center and Research Institutes, Department of Internal Medicine, SoonChunHyang University School of Medicine, Cheonan, Korea

3 Center for Liver Cancer, Hospital, National Cancer Center, Goyang, Korea

4 Department of Internal Medicine, Gangnam Severance Hospital, Yonsei University College of Medicine, Seoul, Korea

submitted 20.9.2018

accepted after revision 16.1.2019

\author{
Bibliography \\ DOI https://doi.org/10.1055/a-0887-4200 | \\ Endoscopy International Open 2019; 07: E792-E795 \\ (c) Georg Thieme Verlag KG Stuttgart · New York \\ eISSN 2196-9736
}

Corresponding author

Jong Ho Moon, MD, PhD, SoonChunHyang University School of Medicine, Digestive Disease Center and Research Institute, SoonChunHyang University Bucheon Hospital, 170 Jomaru-ro, Wonmi-gu, Bucheon, 14584, Korea

Fax: +82-32-621-5080

jhmoon@schmc.ac.kr

\section{ABSTRACT}

Background and study aims Duodenal perforation by migration of plastic stents placed to treat biliary lesions is rare but can be life-threatening. Surgical management is preferred, but it may increase risks of mortality and morbidity, especially in patients with underlying comorbidities and those of advanced age. We describe five cases of duodenal perforation that were successfully managed endoscopically. Four patients were elderly, and one had endstage renal disease. We used cylindrically adapted cap-fitted endoscopy to successfully retrieve migrated plastic stents and to close the perforated walls with hemoclips. No post-procedural complication was noted. In conclusion, endoscopic management is appropriate as a first-line approach in patients with duodenal perforations caused by plastic stent migration.

\section{Introduction}

Endoscopic biliary drainage (EBD) using a plastic stent (PS) is frequently performed during endoscopic retrograde cholangiopancreatography (ERCP) to resolve biliary obstructions [1]. Duodenal perforation due to stent migration is rare but can be life-threatening. Surgical management is the standard treatment for iatrogenic duodenal perforation.

We report successful endoscopic management of patients with duodenal perforation secondary to migrated PS ( $\triangleright$ Table 1).

\section{Cases reports}

\section{Patient 1}

A 50-year-old male with end-stage renal disease presented with diffuse abdominal pain. He had undergone EBD using a PS (CLSO, $10 \mathrm{Fr}, 10 \mathrm{~cm}$; Cook Endoscopy, Winston-Salem, North Carolina, United States) 4 weeks prior for treatment of a biliary leak after laparoscopic cholecystectomy. The patient had a distended abdomen and diffuse abdominal tenderness, and an abdominal $x$-ray showed distal migration of the PS. Abdominal computed tomography (CT) demonstrated that the PS had penetrated the peritoneal cavity through the duodenal wall ( $>$ Fig.1a). Endoscopic findings showed a duodenal fistula, and the proximal portion of the PS was almost penetrating the duodenal wall ( $\triangleright$ Fig. 1b). Endoscopic retrieval of the migrated 
- Table 1 Summary of cases.

\begin{tabular}{|c|c|c|c|c|c|c|c|}
\hline $\begin{array}{l}\text { Case, } \\
\text { no. }\end{array}$ & $\begin{array}{l}\text { Age/ } \\
\text { sex }\end{array}$ & $\begin{array}{l}\text { Chief } \\
\text { complaint }\end{array}$ & $\begin{array}{l}\text { Indication } \\
\text { for EBD }\end{array}$ & $\begin{array}{l}\text { Location of } \\
\text { malignant } \\
\text { stricture }\end{array}$ & $\begin{array}{l}\text { Stent no. } \\
\text { Diameter/length } \\
\text { (name, company) }\end{array}$ & $\begin{array}{l}\text { Intervals from } \\
\text { stent insertion to } \\
\text { perforation (Days) }\end{array}$ & $\begin{array}{l}\text { Devices used for } \\
\text { retrieving PS and } \\
\text { closing perforated wall }\end{array}$ \\
\hline 1 & $50 / M$ & $\begin{array}{l}\text { Abdominal } \\
\text { pain }\end{array}$ & $\begin{array}{l}\text { Biliary leak } \\
\text { after laparo- } \\
\text { scopic chole- } \\
\text { cystectomy }\end{array}$ & - & $\begin{array}{l}1 \\
10 \mathrm{Fr} / 10 \mathrm{~cm} \\
\text { (CLSO, Cook) }\end{array}$ & 28 & $\begin{array}{l}\text { Transparent cap } \\
\text { Snare } \\
\text { Hemoclips }\end{array}$ \\
\hline 2 & $78 / F$ & $\begin{array}{l}\text { Fever } \\
\text { Abdominal } \\
\text { pain }\end{array}$ & GB cancer & $\begin{array}{l}\text { Proximal } \\
\text { CBD }\end{array}$ & $\begin{array}{l}2 \\
7 \mathrm{Fr} / 10 \mathrm{~cm}, 12 \mathrm{~cm} \\
(\mathrm{CLSO}, \text { Cook) }\end{array}$ & 3 & $\begin{array}{l}\text { Transparent cap } \\
\text { Rat-tooth forceps } \\
\text { Hemoclips }\end{array}$ \\
\hline 3 & $72 / M$ & $\begin{array}{l}\text { Abdominal } \\
\text { pain }\end{array}$ & $\begin{array}{l}\text { Klatskin tu- } \\
\text { mor, Bismuth } \\
\text { type Illa }\end{array}$ & $\begin{array}{l}\text { Proximal } \\
\text { CBD }\end{array}$ & $\begin{array}{l}1 \\
10 \mathrm{Fr} / 12 \mathrm{~cm} \\
\text { (Double Layer, } \\
\text { Olympus) }\end{array}$ & 2 & $\begin{array}{l}\text { Transparent cap } \\
\text { Snare } \\
\text { Hemoclips \& fibrin glue }\end{array}$ \\
\hline 4 & $84 / F$ & $\begin{array}{l}\text { Abdominal } \\
\text { pain }\end{array}$ & $\begin{array}{l}\text { Klatskin tu- } \\
\text { mor, Bismuth } \\
\text { type Illa }\end{array}$ & $\begin{array}{l}\text { Proximal } \\
\text { CBD }\end{array}$ & $\begin{array}{l}1 \\
10 \mathrm{Fr} / 12 \mathrm{~cm} \\
\text { (CLSO, Cook) }\end{array}$ & 21 & $\begin{array}{l}\text { Snare } \\
\text { Hemoclips }\end{array}$ \\
\hline 5 & $73 / F$ & $\begin{array}{l}\text { Jaundice } \\
\text { Abdominal } \\
\text { pain }\end{array}$ & GB cancer & $\begin{array}{l}\text { Proximal } \\
\text { CBD }\end{array}$ & $\begin{array}{l}1 \\
10 \mathrm{Fr} / 15 \mathrm{~cm} \\
\text { (CLSO, Cook) }\end{array}$ & 51 & $\begin{array}{l}\text { Rat-tooth forceps, } \\
\text { alligator-jaw rotatable } \\
\text { grasping forceps } \\
\text { Hemoclips }\end{array}$ \\
\hline
\end{tabular}

PS was planned. The proximal flap of the PS was snared under fluoroscopic guidance, and a snare was inserted into the fistula to retrieve the PS. The perforated lesion was closed with hemoclips under cylindric adapter cap-fitted endoscopy ( $\downarrow$ Fig.1c, - Video 1).

The patient fasted for 3 days, during which no leak in the upper gastrointestinal tract was detected using water-soluble contrast medium.

\section{Patient 2}

A 78-year-old female underwent EBD using two straight PSs (CLSO, $7 \mathrm{Fr}, 10$ and $12 \mathrm{~cm}$, Cook Endoscopy) to treat a biliary stricture caused by gallbladder (GB) cancer. Three days later, the patient complained of high fever $\left(38.9^{\circ} \mathrm{C}\right)$ and abdominal pain. Abdominal CT showed a duodenal perforation.

ERCP revealed a migrated biliary stent. A small, round ulcer with a perforated wall was observed in the second portion of the duodenum. The migrated stent was retrieved using rattooth forceps. Hemoclipping was performed to close the perforated lesion. The patient recovered completely.

\section{Patient 3}

A 72-year-old male underwent biliary drainage using a PS (Double Layer, $10 \mathrm{Fr}, 12 \mathrm{~cm}$; Olympus, Tokyo, Japan) for palliation of Klatskin tumor, Bismuth type IIla. After 2 days, he complained of right upper and lower quadrant pain with tenderness. CT revealed distal stent migration and duodenal perforation.

Under cylindric adapter cap-fitted endoscopy, the distally migrated stent in the second portion of the duodenum was removed and the perforated lesion was closed using hemoclips and fibrin glue (Green Plast; Green Cross, Young-in, Korea). The PS was exchanged for a metal stent after 2 weeks.

\section{Patient 4}

An 84-year-old female underwent ERCP and EBD using a PS (CLSO, $10 \mathrm{Fr}, 12 \mathrm{~cm}$, Cook Endoscopy) 3 weeks prior for palliation of a Klatskin tumor, Bismuth type Illa. The patient complained of constant mild abdominal pain after EBD. CT revealed PS penetrating the opposite side of the duodenal wall into the peritoneal cavity. The distal tip was surrounded by an area of fluid collection $5 \mathrm{~cm}$ in diameter.

Duodenoscopy confirmed a duodenal perforation and distal migration of the PS. The perforated duodenal wall was closed with hemoclips after stent removal, and the collected peritoneal fluid was drained externally. After draining $90 \mathrm{~mL}$ bilious peritoneal fluid, the catheter was removed. The patient tolerated a solid diet for 4 days after removal of the PS.

\section{Patient 5}

A 73-year-old female with biliary stricture due to GB cancer underwent EBD using a PS (CLSO, 10 Fr, 15 cm; Cook Endoscopy). The patient was readmitted 51 days later because of recurrent jaundice and abdominal pain. Distal migration of the PS and duodenal perforation were revealed by CT. The PS was removed using rat-tooth and alligator-jaw rotatable grasping forceps (FG-44-NR-1; Olympus, Tokyo, Japan), the perforated duodenal wall was closed using hemoclips, and a percutaneous transhepatic biliary drainage was inserted. One week later, no evidence of leakage in the upper gastrointestinal tract was detected using water-soluble contrast medium. 

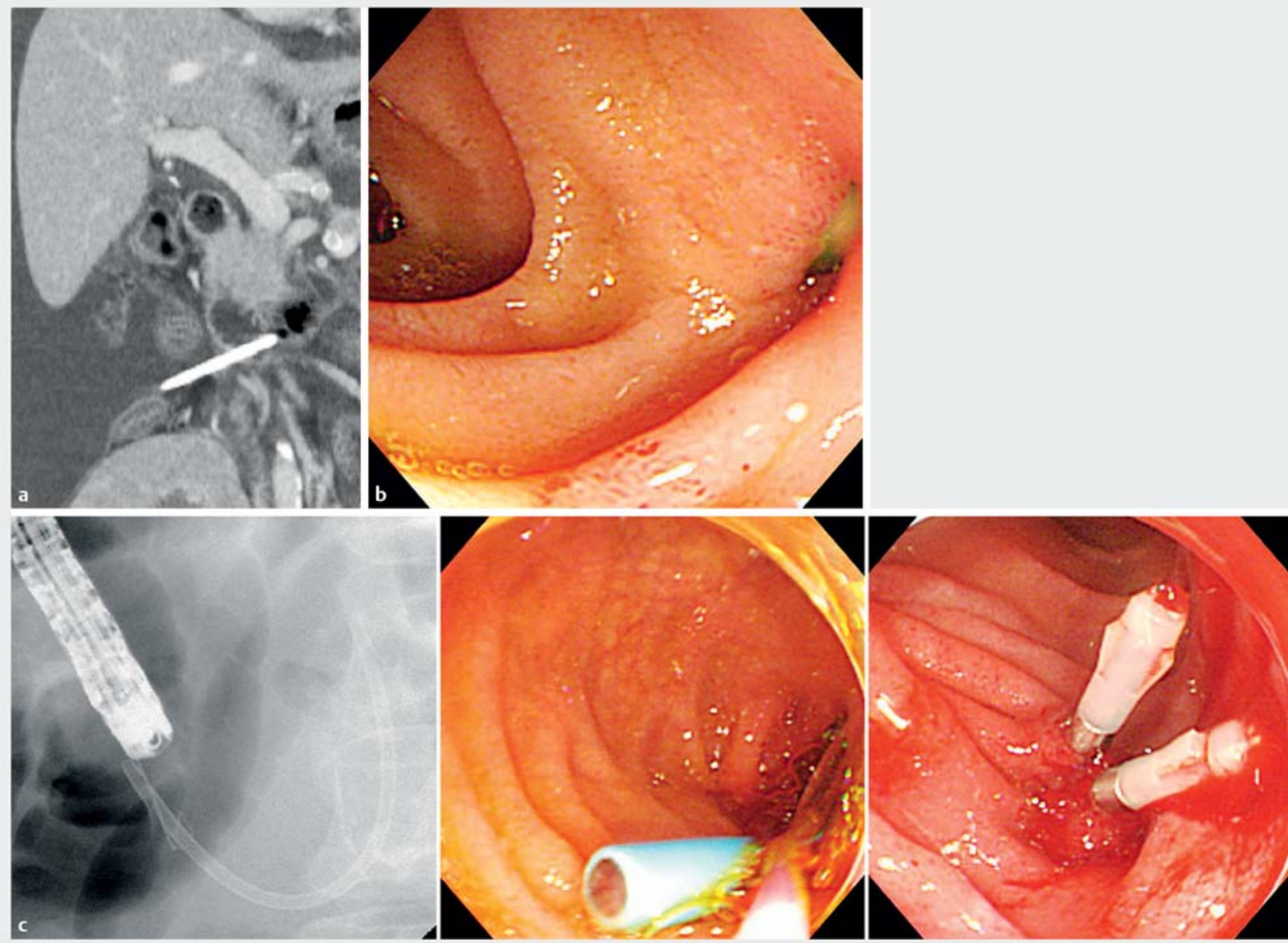

- Fig. 1 a CT showing downward migration of a biliary plastic stent and perforation of the lateral wall of the second loop of the duodenum. b A small portion of the proximal part of the migrated plastic stent and perforation detected during endoscopy. c The migrated plastic stent was retrieved by endoscopic snaring. The perforation was closed successfully with hemoclips by cylindric adapter cap-fitted endoscopy.

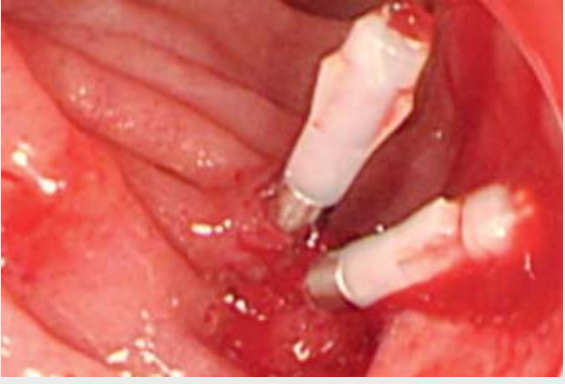

$\checkmark$ Video 1 Duodenoscopy findings showed a perforated duodenal fistula with proximal tip of migrated plastic stent. The plastic stent was almost penetrating the duodenal wall. The proximal part of the migrated plastic stent was visible on the duodenal lumen after the cap was pushed during endoscopy. The perforated hole was closed with hemoclips after retrieval of the plastic stent with a snare.

\section{Discussion}

EBD using a PS is typically performed during ERCP to treat or palliate biliary obstruction or leakage [2]. This is a safe procedure with minimal adverse effects.

Early complications of EBD, including stent migration, occur within 2 weeks after EBD in approximately $5 \%$ of patients. Most late complications of biliary stenting are associated with stent migration. The migration rates of PSs are reportedly $3.1 \%$ to $4.9 \%$ and $3 \%$ to $6 \%$ in the proximal and distal directions $[1,3]$. Migration of PS is more frequent in patients with benign than with malignant biliary strictures, particularly hilar strictures [4], and with single than with multiple stents [3]. This may be because benign stenosis is relieved after recovery of inflammation, while malignant stenosis can increase in size and anchor securely. No particular type, size, or caliber of stent has been implicated in stent-related perforations [1].

All migrated stents were inserted proximal of the stricture. Johanson et al. reported that the migration occurred in $5 \%$ to $7.5 \%$ of patients even though proper placement of the stent in the desired duct was verified both endoscopically and fluoroscopically before completion of the procedure $[5,6]$. Most PS are 
spontaneously eliminated after distal migration. Intestinal perforation is a rare but serious adverse event. Biliary stents can cause duodenal perforations by any of the following mechanisms. First, the stent may be placed incorrectly, leading to irritation and, eventually, necrosis of the duodenal mucosa. Second, inflexibility or incorrect length of the stent may irritate the duodenal mucosa and lead to pressure necrosis. Third, adhesion of the tumor to the proximal portion of a PS may increase the intensity of trauma caused by the distal intraduodenal segment of the PS, preventing its adaption to intestinal peristalsis [2].

Not all patients with duodenal perforation complain of typical symptoms of intestinal perforation. A greater than 24-hour delay in diagnosis adversely affects the treatment outcome and prognosis [2]; therefore, early diagnosis and proper management are crucial in such cases. Plain abdominal radiography can reveal stent position and migration. CT is the modality of choice for detecting perforations and locating the migrated stent [2].

Surgical stent removal and closure of the perforation are the mainstays of treatment for intestinal perforation [7]. However, endoscopic management of iatrogenic perforation in a clinically stable patient is now justified due to improvements in endoscopic techniques [2]. Indeed, several cases have been reported of duodenal perforation successfully treated with hemostatic clipping and fibrin glue under endoscopy [7 - 10].

Determining appropriate management of biliary stent migration-induced perforation requires knowledge of the location of the perforation, its containment in the peritoneal or retroperitoneal space, and the patient's clinical presentation. If the perforation is small and located in the retroperitoneal space, conservative management with antibiotics may be effective. However, if the perforation is uncontained on the lateral wall of the duodenum, or if peritonitis or other life-threatening symptoms are present, aggressive surgical intervention is required. Patient 1 complained of signs of peritoneal irritation, and endoscopy showed a duodenal fistula almost penetrating the duodenal wall, with the PS in the peritoneal cavity. Patient 2 had panperitonitis. Both patients were treated successfully using endoscopic techniques.

Several devices and materials including hemoclips, fibrin glue, and transparent cap can be used to close a perforated duodenal wall. The most common method is endoscopic closure of the perforation using hemoclips. Fibrin glue was used in Patient 3 to ensure complete closure of the perforation. Cap-assisted endoscopy under direct vision was helpful in Patients 1,2 , and 3 , as it provides a better view and enables the curvature of the duodenum to be overcome. And we were able to approximate the duodenal wall to the distal end of the endoscope aided by suction to remove a migrated PS. An over-thescope-clip (OTSC) can be used to close the perforated hole. But it costs far more than hemoclips and the perforated hole caused by PS migration is small enough to close with the hemoclips we used. It would be a treatment option for failure cases after hemoclipping.

Prevention of PS migration is the most important issue; however, conventional PS are rigid and tend to straighten out and migrate. For this reason, PS manufactured from alternative materials have been developed. For example, Cheon et al. reported that a stent made from polyurethane, which is soft and pliable, prevents PS migration, particularly distal migration [6].

\section{Conclusion}

In conclusion, PS migration and duodenal perforation are rare. It is important to detect perforation early, including in patients without typical signs of panperitonitis. Endoscopic retrieval of migrated biliary stents and closure of duodenal perforations are feasible in clinically stable patients. This would be useful in elderly cancer patients with comorbidities, which can exacerbate postoperative morbidities and increase mortality.

\section{Acknowledgement}

This study was supported in part by the SoonChunHyang University Research Fund.

\section{Competing interests}

None

\section{References}

[1] Sinha S, Wardle A, Kalidindi V et al. Erosion and perforation of the biliary tree by plastic biliary endoprostheses. Endoscopy 2010; 42: $760-763$

[2] Saranga Bharathi R, Rao P et al. latrogenic duodenal perforations caused byendoscopic biliary stenting and stent migration: an update. Endoscopy 2006; 38: $1271-1274$

[3] Dumonceau JM, Tringali A, Blero D et al. Biliary stenting: indications, choice of stents and results: European Society of Gastrointestinal Endoscopy (ESGE) clinical guideline. Endoscopy 2012; 44: 277-298

[4] Perdue DG, Freeman ML, DiSario JA et al. Plastic versus self-expanding metallic stents for malignant hilar biliary obstruction: a prospective multicenter observational cohort study. J Clin Gastroenterol 2008; 42: $1040-1046$

[5] Johanson JF, Schmalz M], Geenen JE. Incidence and risk factors for biliary and pancreatic stent migration. Gastrointest Endosc 1992; 38 : $341-346$

[6] Cheon YK, Oh HC, Cho YD et al. New 10F soft and pliable polyurethane stents decrease the migration rate compared with conventional $10 \mathrm{~F}$ polyethylene stents in hilar biliary obstruction: results of a pilot study. Gastrointest Endosc 2012; 75: 790 - 797

[7] Mutignani M, lacopini F, Dokas S et al. Successful endoscopic closure of a lateral duodenal perforation at ERCP with fibrin glue. Gastrointest Endosc 2006; 63: $725-727$

[8] Roses LL, Ramirez AG, Seco AL et al. Clip closure of a duodenal perforation secondary to a biliary stent. Gastrointest Endosc 2000; 51: 487-489

[9] Lee TH, Bang BW, Jeong Jl et al. Primary endoscopic approximation suture under cap-assisted endoscopy of an ERCP-induced duodenal perforation. World J Gastroenterol 2010; 16: 2305-2310

[10] Prachayakul V, Aswakul P, Kachintorn U. Duodenal perforation due to plastic stent migration successfully treated by endoscopy. Gastrointest Endosc 2012; 75: 1265 - 1266 